La forme typique et les variétés de cette espèce peuvent se distinguer de la façon suivante :

1. Tète testacée.........................

2. Prothorax et base des élytres plus ou moins foncés ou enfu-

més (diluta Ach.) .................. argentina Pic.

2'. Prothorax et base des élytres testacé-rougeâtre concolore.

$\ldots \ldots \ldots \ldots \ldots \ldots \ldots \ldots \ldots \ldots \ldots$. tucumana $n$. var.

1'. Tète et prothorax noirs, concolores........ v. bicolor Ach.

La forme type et la v. bicolor Ach. proviennent de Buenos-Ayres, tandis que la v. tucumana Pic est originaire du Tucuman.

\title{
Diagnoses de Galerucini nouveaux d'Afrique
}

[Col. Chrysomelidae] (1)

par V. LABOISSIÈRE.

Stenella limbata, n. sp. - Allongé subparallèle, testacé brunâtre, vertex et élytres noir brillant, les derniers bordés de brunâtre sur le bord latéral dans toute sa longueur, surface très finement ponctuée; dessous testacé, abdomen noir, dernier segment jaune; pattes testacé brunâtre, derniers articles des tarses plus foncés. - Long. 4-4,כ̋ mm.

Afrique Orientale : Tavéta [alt. $750 \mathrm{~m}$.] (Alluadd et Jeannal, mars 1912). Muséum de Paris et coll. Laboissière.

Gerochroa cincta, n. sp. - Ovale un peu allongé, noir, tète et pronotum testacé roussâtre, la première avec une tache sur le vertex, le second cinq taches peu apparentes, noires; surface grossement ponctuée, antemnes noires, écusson brun roux, élytres noir verdâtre complètement bordés de roux, plus fortement en arrière et au sommet, densément et fortement ponctués. Dessous et pattes noirs, processus métasternal conique s'avançant jusqu'au niveau des hanches antérieures. - Long. $14 \mathrm{~mm}$.

Afrique occidentale : Congo français : Mayoumba. Coll. Laboissière.

Gandezea marangana, n. sp. - Ovale oblong, jaune testacé faiblement rougeâtre sur le pronotum et les cuisses, labre et poitrine

(1) Cf. Bull. Soc. ent. Fr. [1917], p. 327-329; - l. c. [1919], p. 281-283; - l. c. [1919], p. $302-305 ;-l$. c. $\lfloor 1919]$, p. $329-332 ;-l . c .[1919]$, p. $365-$ 368 , 
noirs ainsi que l'extrême base du premier article des tarses postérieurs et le dernier article des antennes. - Long. 6,כ -7 mm.

Afrique Orientale : Fort-Hall dans le district de Maranga sur le versant sud-ouest du mont Kénia (Alluaud, nov. 1904). Muséum de Paris et coll. Laboissière.

Candezea rostrata, n. sp. - $\sigma^{\pi}$. Jaune brillant, plus pâle sur les élytres; hanches, cuisses, poitrine et abdomen, moins le sommet du quatrième segment et le cinquième entièrement jaunes, noir brillant; écusson également noir, lisse. Tête à face convexe sans aucune carène. - Long. 4,25 $\mathrm{mm}$.

Afrique Orientale : zone inférieure du mont Kilimandjaro (AlLuAdD, janv.-avril 1904).

Gandezea umbilicata, n. sp. - Ovale, jaune testacé, un point à la base du premier article des tarses postérieurs, sommet des mandibules, dernier article des antennes et trois taches sur les élytres noirs, ces taches placées : la première transversale près de la base, les deux autres transversalement après le milieu; surface convexe, couverte de gros points profonds dont le diamètre est plus grand que les intervalles qui les séparent; au fond, vu à un fort grossissement, on aperçoit deux ou trois petits points brillants. - Long. 4,7 ว $\mathrm{mm}$.

Afrique Orientale : Mombasa (Alluadd, juillet 1904). Muséum de Paris.

Gandezea clathrata, n. sp. - Elliptique, jaune testacé, vertex rougeâtre, pronotum faiblement verdâtre, antennes rembrunies à partir du quatrième article, élytres avec une bande latérale, la base, le sommet et la suture noirs et en plus six bandes longitudinales parallèles, brunes, peu marquées, n'atteignant ni la base ni le sommet, plus visibles en arrière. - Long. 4,כ mm.

Afrique Orientale : de Tiwi à Gazi (Alluaud et Jfannel, nov. 1911). Muséum de Paris.

Monolepta Alluaudi, n. sp. - Tète, antennes, pronotum et pattes en majeure partie jaune pâle, élytres, poitrine et abdomen rougeâtres. - Long. 4,כ̆-כ mm.

Afrique Orientale : Kijabi [alt. $2.100 \mathrm{~m}$.] (Alluaud et Jeannel, déc. 1911); - versant ouest du mont Kénia dans la zone des forêts inférieures [alt. $2.400 \mathrm{~m}$.] (AlLuaud et JEANNEL, janv.-février 1912); Pori de Mbuyuni [alt. 1.110 m.] (Alluadd et Jeannel, mars 1912); - Landiani [alt. 2.500 m.] (Alluadd, oct. 1904). Muséum de Paris et coll. Laboissière.

Monolepta Jeanneli, n. sp. - Tête, pronotum et partie des 
cuisses noirs, antennes, tibias et tarses flaves, poitrine, abdomen et èlytres rouges, les derniers avec un fin liséré noir sur la moitié antérieure du bord latéral. — Long. 5 mm.

Alrique Orientale : Kijabé [alt. 2.100 m.] (Alluadd et Jeannel, déc. 1911). Muséum de Paris.

Monolepta Jeanneli var. dimidiata, n. var. - Coloration de l'espèce typique, mais l'écusson et le tiers basal des élytres noirs, cuisses postérieures noires sauf au sommet. - Long. כ̃ mm.

Afrique Orientale : Rivière Ambony dans la zone inférieure du mont Kénia (Alluadd 1909). Muséum de Paris.

Monolepta Jeanñeli var. bicincta, n. var. - Diffère de la var. dimidiata par une deuxième bande large, noire, placée après le milieu de l'élytre et reliée à la bande basale par une ligne noire longeant le bord latéral, écusson rougeâtre. - Long. $4 \mathrm{~mm}$.

Afrique Orientale : Ouganda central dans la région de Boujongolo (Alluaud, décembre 1909).

Monolepta ugandaensis, n. sp. - Ovale, rougeâtre, abdomen jaune, côtés du prosternum, partie antérieure de la tête, marge latérale du pronotum et deux bandes transversales, la première basale, la seconde postmédiane, sur chaque élytre, noires. - Long. 3,75 mm.

Afrique Orientale : Unyoro méridional (ALLUAUd, janvier 1909). Muséum de Paris.

Monolepta ephippiata Gerst. var. keniaensis, n. var. - Tête à part quelques points jaunes vers l'épistome et pronotum noirs, écusson noir; élytres entièrement bordés de noir sur le bord latéral, la suture, la base et le sommet avec, en outre, la bande onduleuse transversale, placée un peu avant le milieu, qui existe chez l'espèce typique. - Long. כั- ว, כ̆ mm.

Afrique Orientale : Voï [alt. $600 \mathrm{~m}$.] (Alluaud et Jeannel, mars 1911); - Rivière Ambony dans la zone inférieure du mont Kenia (Alluadd, nov. 1909). Muséum de Paris et coll. Laboissière.

Monolepta buraensis, n. sp. - Jaune testacé, partie antérieure de la tête noire, antennes pâles avec la pointe du dernier article noire, écusson brun roux. Élytres avec une bande basale noire, parfois remplacée par deux taches, l'une juxtascutellaire, l'autre humérale, reliée latéralement à une bande médiane transversale, une troisième bande arquée, noire, avant le sommet, remontant légèrement sur le bord latéral et limitée en arrière par une ligne rougeâtre qui longe toute la suture et rejoint latéralement la bande médiane. Dessous jaune testacé, poi- 
trine et cuisses postérieures rougeâtres, pygidium noir. - Long. כั-

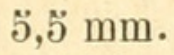

Afrique Orientale : Bura [alt. 1.0כั0 m.] (Alluaud et Jeannel, mars 1912); - Voï (Alluaud sept. 1907). Muséum de Paris et coll. Laboissière.

Monolepta deleta Weise, var. bambusae, n. var. - Dessous, pattes, antennes et écusson noirs, tête et pronotum jaune rougeâtre, élytres entièrement jaune testacé ou jaune pâle avec parfois une faible ligne rougeâtre partant du calus et s'étendant sur le bord latéral. - Long. 3,̋̈-4,כ̆ mm.

Afrique Orientale : monts Arberdare, de Nyéré à Naivasha, forêts de bambous sur le versant ouest du mont Kinangop [alt. 2.700-3.000 m. (Alluaud et Jeannel, février 1912).

\section{Un Membracide [Hem.] nouveau pour la Faune française}

\section{par le $\mathrm{D}^{\mathrm{r}} \mathrm{V}$. LaLLemand.}

Durant mon séjour à Montpellier en qualité de médecin attaché à l'hòpital militaire, M. E. Lavagne me confia la détermination des $\mathrm{Ho}_{0}$ moptères qu'il avait recueillis dans la région. Je fus très surpris de rencontrer parmi ceux-ci des Membracides du genre Ceresa, genre dont aucun représentant n'a encore été signalé en Europe. Ces insectes, que je rapportai à Ceresa bubalus F., avaient été récoltés à St-Guilhem-le-Désert et à Lattes (Hérault). M. Lavagne ne put me donner aucune autre indication sur leur capture mais, en septembre 1918 , j'ai recueilli moi-même à Lattes, en fauchant sur des Equisetum parmi lesquels serpentaient des tiges de jeunes vignes, trois individus de cette espèce.

Ceresa bubalus F. est répandu aux États-Unis, principalement dans la région orientale (Colorado, Utah, Californie); il est donc possible que cette espèce ait été importée en Europe avec des jeunes plants de vigne, bien qu'il soit curieux de la rencontrer à Lattes et à $\mathbf{S}^{\mathrm{t}}$-Guilhem, deux régions absolument dissemblables et très distantes. 


\section{$2 \mathrm{BHL}$ Biodiversity Heritage Library}

Laboissière, V. 1920. "Diagnoses de Galerucini nouveaux d'Afrique [Col. Chrysomelidae]." Bulletin de la Société entomologique de France 1920, 50-53.

View This Item Online: https://www.biodiversitylibrary.org/item/34136

Permalink: https://www.biodiversitylibrary.org/partpdf/119043

\section{Holding Institution}

Smithsonian Libraries

\section{Sponsored by}

Smithsonian

\section{Copyright \& Reuse}

Copyright Status: NOT_IN_COPYRIGHT

This document was created from content at the Biodiversity Heritage Library, the world's largest open access digital library for biodiversity literature and archives. Visit BHL at https://www.biodiversitylibrary.org. 